\title{
Developing a Measurement Scale for 7-S Framework
}

\author{
Rajan Pothiyadath $^{1}$, Dr. J. Reeves Wesley ${ }^{2}$ \\ ${ }^{1}$ Research Scholar, Karunya University, Coimbatore, India. \\ ${ }^{2}$ Professor, VIT Business School, Chennai, india
}

\begin{abstract}
Organisations used every possible methods and strategies to gain competitive advantage in the market place. One such strategy involves gaining internal strength using the human resources of the organisation. Many methods have been evolved in this context; however, a composite framework has been developed by McKinsey called the 7-S framework. However, a prior review of literature indicates that no fit scale is available to measure the implementation of 7-S framework. This paper makes an attempt to develop a measurement scale on 7-S framework on the public sector undertaking company, the Kerala State Electricity Board (India).
\end{abstract}

\section{Introduction}

The McKinsey 7-S framework was developed by McKinsey consultants, including Tom Peters and Robert H. Waterman, with the academic partnership of Richard Pascale and Anthony G. Athos in the early 1980s. Many other frameworks have been developed by different authors involving the traditional factors such as the traditional mass production tangibles of capital, infrastructure, and equipment, however, no single model has been evolved that emphasises human resources as major cause of performance in a changed environment. The 7-Ss are: Structure, Strategy, Skills, Staff, Style, Systems, and Shared values.

Strategy: The coherent set of action by which a company plans to achieve competitive advantage Style: The tangible patterns of evidence regarding the priorities of the top management team

Skills: A synergistic blending of dominating attributes and capabilities in the Corporation

Systems: The routine procedural processes and flows of ordinary operations

Structure: The formal assignment of organizational specializations, authority, and responsibility

Staff: People in organization, considered in terms of corporate demographics, not individual personalities

Shared Values: The guiding concepts and vision underlying the desired corporate destiny

At inception, this framework invited criticism on its ability to affect organisational effectiveness. The argument was centred on the other authors contention on certain elements of 7-S especially such as structure - who does what, who reports to whom, and the like, but, a composite framework that include the constituents of 7-S has not been studied. An understanding of 7-S is now very imperative as organizations grew in size and complexity and the more critical question became one of human coordination.

It is understood that there is no hierarchy in working on each part of the framework, however, implementation of one would have an effect on the other. So, the success on each part of the framework has to be cautiously achieved. This framework is over 30 years old and many organisations draw cue from each part and believe that organisation is not any part of the framework in isolation, but a composite one which is common to any level of the organisation.

The increase in domestic and foreign competition, in particular, with the advent of multi-national companies in the Indian soil has brought several changes in the demography and a transformation in the way companies operate and compete. Earlier, public sector companies were overly protected by the Governments at the State and the National level and they enjoyed monopoly in the market without competition. But now an overplay of competition has set in partially or completely and public sector companies operate in an open market where competition rules the roost. To meet this new paradigm, these firms are employing established norms of the private sector in order to stay fit. More essentially, on the HR front more adaptive measures have been undertaken so that they remain fit and robust. Or in other words, they employ flexible physical, human, and organizational resources to increase the probability of sustaining a competitive advantage in a hypercompetitive environment (Parthasarthy \& Sethi, 1992 \& 1993).

This paper does not make an attempt to develop a conceptual framework involving the constituents of the 7-S framework, but to develop a measurement scale to measure the 7 constituents of the 7-S framework in a Government company in the power sector, the Kerala State Electricity Board (KSEB). Though the power sector reforms literally started in distribution sector in the year 1998, the actual momentum picked up after parliament passed the bill in 2003 and the Honourable president of India approved it on June $8^{\text {th }} 2003$. But even after elapse of a decade Kerala is one of the states still hesitant to bring in the stipulated changes. 
Moreover, the authors have chosen this sector to develop a scale for the implementation of 7-S framework because power sector in India is undergoing serious transformation and structural changes across India. Hence, the major challenges considering the present scenario in KSEB representing the 7 constituents were developed as items and subjected to validity and reliability check.

\section{Methodology}

For the purpose of studying the objectives and testing the hypotheses, a questionnaire was used as an instrument to collect the data.

The items capturing each factor in the 7-S framework were adapted from standardized questionnaires developed or used by earlier researchers. However, they were subjected to validity and reliability tests. Hence, the items that constituted adequate coverage of the factors under study were decided and agreed upon by the researcher. The questionnaire was subjected to face and content validity whose determination was judgemental. There are two schools of thought on the distinctiveness of face and content validity. The first one saw face validity as just an indirect approach to the measurement of content validity (Carmines, \& Zeller, 1979; Nunnally, 1970) whereas the second one treated them as separate and different tests (DeVellis, 1991; Kerlinger, 1973). In this study, the researcher has subscribed to the second perspective where quantitative assessment of the content validity has been followed.

\section{Content Validity}

The face and content validity was conducted with 8 experts. The experts scrutinised the items, according to the definition generated against the constructs of $7 \mathrm{~s}$ model such as the structure, strategy, system, skill, staff, styles and shared values. Before they offered their opinion on the items, the researcher informed them of the objectives and the need for the study and then encouraged to express the validity of each item in capturing the adequate information required for the study. Then they were requested to offer their feedback on each of the items. Based on their feedback, few items were slightly modified, reworded, repositioned to make them appropriate for use. The experts also suggested a 5-point rating scale on all possible items. The content validity ratio (CVR) was applied to each item, using the formula developed by Lawsche (1975).

$$
\begin{aligned}
& \text { Content Validity Ratio }(\mathrm{CVR})=\mathrm{Ne}-\mathrm{N} / 2 \\
& \mathrm{~N} / 2 \\
& \text { where } \mathrm{Ne}=\text { number of panellists indicating "essential" } \\
& \text { and } \mathrm{N}=\text { total number of panellists. }
\end{aligned}
$$

\begin{tabular}{|c|c|c|}
\hline No & Items & CVR \\
\hline 1 & Structure of KSEB is suitable to undertake changes towards a Professional Organization. & 0.75 \\
\hline 2 & Strategy of KSEB is fit for an organization looking for Total Customer satisfaction & 0.75 \\
\hline 3 & KSEB's manpower is highly qualified and specialized in skills & 1.00 \\
\hline \multirow[t]{2}{*}{4} & Talents are not attracted to KSEB due to various reasons & 0.75 \\
\hline & & 1.00 \\
\hline 5 & KSEB is utilizing their resources (Men, Money, Materials and Time) optimally. & \\
\hline 6 & Employees of KSEB are loyal and accountable. & 1.00 \\
\hline 7 & KSEB owns a positive business culture suitable for a modern organization & 1.00 \\
\hline 8 & Employees of KSEB shows Team spirit & 1.00 \\
\hline 9 & All decision makers are ready to take some amount of Risk while taking decisions & 1.00 \\
\hline 10 & Technologies used in KSEB are updated regularly. & 1.00 \\
\hline 11 & People are reluctant to join KSEB due to fear of Insecurity. & 1.00 \\
\hline 12 & Skills of employees are right to undertake the job that they are assigned. & 1.00 \\
\hline 13 & Right strategy at Right time is adopted by KSEB in achieving its Vision. & 1.00 \\
\hline 14 & Structure of KSEB is so rigid that it can hamper the progress of Change. & 1.00 \\
\hline 15 & Decentralized or Networked structure would be more appropriate for KSEB. & 1.00 \\
\hline 16 & KSEB takes more time to formulate a right strategy and hence mostly it may not hit the nail. & 0.75 \\
\hline 17 & Skills of employees are not updated in right time to cope with changing environment. & 1.00 \\
\hline 18 & Candidates recruited in KSEB are having competency required for the job. & 1.00 \\
\hline 19 & Technology and systems in KSEB obsolete mostly. & 1.00 \\
\hline 20 & Actions and behaviour of employees focuses customer always. & 1.00 \\
\hline 21 & Employees owns the pride of working with KSEB & 0.75 \\
\hline 22 & Organizational culture of KSEB depicts professionalism. & 1.00 \\
\hline 23 & Decision makers looks for efficiency and economy while making decisions. & 1.00 \\
\hline 24 & System change and business process innovations are regular agenda in KSEB. & 1.00 \\
\hline 25 & Human resources in KSEB are just enough to cater the needs of customer. & 1.00 \\
\hline 26 & Right people with right skills are employed in KSEB & 1.00 \\
\hline
\end{tabular}

The CVR values are given in the below table.

Table showing the Content Validity Ratio (CVR) for the 7-S framework 


\begin{tabular}{lcc}
$\mathbf{2 7}$ & & \\
$\mathbf{2 8}$ & Innovative/Break through strategy is very much adopted in this organization. & 1.00 \\
$\mathbf{2 9}$ & Hierarchical organization structure is a limitation, which delays decision making. & 1.00 \\
$\mathbf{3 0}$ & Structure of KSEB does not follow the strategy to facilitate its vision & 1.00 \\
\hline $\mathbf{3 1}$ & Strategies of KSEB are not fit for a modern and dynamic organization. & 1.00 \\
$\mathbf{3 2}$ & Job design, Job re-engineering, job rotation, HRD etc have made very little progress & 1.00 \\
$\mathbf{3 3}$ & Productivity of staff and officers less compared to a profession style. & 0.75 \\
$\mathbf{3 4}$ & Bench marking of a system is not carried out and used in the system. & 1.00 \\
$\mathbf{3 5}$ & Still employees of KSEB are following a bureaucratic approach in general. & 1.00 \\
\hline
\end{tabular}

All those items which have scored less than 0.50 on the content validity ratio have been removed from the study. Based on the face validity and content validity ratio, the final number of items in each of the factors taking part in this study was decided.

\section{Pilot study and reliability test}

After finalising the number of items in the research instrument using face and content validity tests, a pilot study was undertaken to assess the reliability of the research instrument constructed. To conduct the pilot study, it was decided to select 300 stakeholders without any category size but who had a stake with KSEB at least 2 years as the sampling frame for the pilot study. Each stakeholder was administered with the questionnaire. 300 questionnaires were distributed to the stakeholders and the researcher obtained 240 usable respondents which gives a yield rate of $80 \%$.

The data collected from the pilot study was subjected to reliability test using Cronbach Alpha. It was found that the 7 factors captured using multi-item scales have Cronbach alpha more than 0.7 for all the constituents of the $7 \mathrm{~S}$ framework.

Finally, the questionnaire was standardised with each factor captured with 5 items. The total number of items that stood the validity and reliability check was 35 items. The researcher now conclude that the $7-\mathrm{S}$ framework could be captured using a multi-item scale consisting of 35 items each factor captured with 5 items.

\section{Conclusion}

Change to achieve organisational strategic fit in a new or changed environment may be accomplished through the careful orchestration of the 7-S by the management. The seven factors encompassed by the framework could be implemented thus creating a firm-wide ramification when individual factors are changed. While the configuration of the framework suggests that no single organization factor is omnipotent, however, the relative importance of each factor varies according to the specific context.

\section{References}

[1]. Carmines, E. G., and Zeller, R. A. (1979). Reliability and validity assessment, Newbury Park, CA: Sage Publications.

[2]. DeVellis, R. F. (1991). Scale development: Theory and applications. Applied Social Research Methods Series, 26, Newbury Park, CA: Sage Publications.

[3]. Kerlinger, F. N. (1973). Foundations of behavioral research $\left(2^{\text {nd }}\right.$ Ed.). New York.

[4]. Lawsche, C. H. (1975). A quantitative approach to content validity, Personnel Psychology, 28(4):563-575.

[5]. Nunnally, J.C. (1970). Introduction to psychological measurement. New York: McGraw Hill.

[6]. Parthasarthy, R. and Sethi, S. (1992), 'The impact of flexible automation on business strategy and organizational structure', Academy of Management Review, Vol. 17, nr. 1, p. 86-111.

[7]. Parthasarthy, R. and Sethi, S. (1993), 'Relating strategy and structure to flexible automation: a test of fit and performance implications', Strategic Management Journal, Vol. 14, p. 529-549. 